\title{
Advantage of additive manufacturing in printed board circuits
}

Marlon Roney Delcid, Mechatronic Engeniering ${ }^{1}$, Maria Elena Perdomo, Master's degree in industrial engineering, and Jose Luis Ordoñez, Master's degree in industrial engineering

Universidad Tecnológica Centroamericana, Honduras, maría_perdomo@unitec.edu,jlordonez@unitec.edu

\begin{abstract}
Currently, there is access to a variety of technologies that bring countless benefits to the industry every day, among them we find additive manufacturing, which is recognized worldwide for $3 D$ printing. However, there are different branches or families in which this technology can be classify, this study focuses on the printing of electronic circuits through additive manufacturing. With the aim of identifying the advantages that this technology brings, against traditional technologies such as subtractive manufacturing in electronic circuits. Using a mixed methodology, these advantages are defined by analyzing the machining time as a dependent variable, various tests are carried out using additive manufacturing oriented and a subtractive one, where the most important results are the multiplatform work of the additive manufacturing against their extended machining times, it means, the time of the subtractive processes is approximately $8 \%$ of those employed in the other technology. Finally, it is concluded that additive manufacturing has a variety of advantages but that the use of subtractive technologies is more productive for the industry.
\end{abstract}

Keywords-additive manufacturing (AM), milling, computer numerical control (CNC), revolutions per minute (RPM), 3D printing, subtractive manufacturing, printed circuit board (PCB).

Digital Object Identifier (DOI):

http://dx.doi.org/10.18687/LACCEI2020.1.1.32

ISBN: 978-958-52071-4-1 ISSN: 2414-6390

$18^{\text {th }}$ LACCEI International Multi-Conference for Engineering, Education, and Technology: "Engineering, Integration, and Alliances for a Sustainable Development" "Hemispheric Cooperation for Competitiveness and Prosperity on a Knowledge-Based Economy", 27-31 July 2020, Virtual Edition. 


\title{
Ventaja de la fabricación aditiva en circuitos electrónicos
}

\author{
Marlon Roney Delcid, Mechatronic Engeniering ${ }^{1}$, Maria Elena Perdomo, Master's degree in industrial engineering, \\ and Jose Luis Ordoñez, Master's degree in industrial engineering \\ Universidad Tecnológica Centroamericana, Honduras, maría_perdomo@unitec.edu,jlordonez@unitec.edu
}

\begin{abstract}
Resumen- Actualmente, hay acceso a una variedad de tecnologías que traen innumerables beneficios a la industria todos los días, entre ellos encontramos la fabricación aditiva, que es reconocida mundialmente por la impresión 3D. Sin embargo, hay diferentes ramas o familias en las que se puede clasificar esta tecnología, este estudio se centra en la impresión de circuitos electrónicos a través de la fabricación aditiva. Con el objetivo de identificar las ventajas que aporta esta tecnología, frente a tecnologías tradicionales como la fabricación sustractiva en circuitos electrónicos. Utilizando una metodología mixta, estas ventajas se definen analizando el tiempo de mecanizado como una variable dependiente, se llevan a cabo varias pruebas utilizando una fabricación aditiva orientada y una sustractiva, donde los resultados más importantes son el trabajo multiplataforma de la fabricación aditiva contra su mecanizado extendido veces, es decir, el tiempo de los procesos sustractivos es aproximadamente el $8 \%$ de los empleados en la otra tecnología. Finalmente, se concluye que la fabricación aditiva tiene una variedad de ventajas pero que el uso de tecnologías sustractivas es más productivo para la industria.

Keywords: fabricación aditiva (AM), fresado, control numérico por computadora (CNC), revoluciones por minuto (RPM), impresión 3D, fabricación sustractiva, placa de circuito impreso (PCB).
\end{abstract}

\section{INTRODUCCIÓN}

El avance logrado con la incursión de la manufactura aditiva (AM) a la industria internacional ha sido sin lugar a duda revolucionario, dejando en el pasado los métodos tradicionales y las largas esperas para fabricar una pieza donde se recurría a alguien que tuviera acceso al equipo adecuado para la realización de un trabajo de calidad. Desde su inicio, la AM ha estado dominada por la tecnología independiente lo que quiere decir que ha sido desarrollada en distintos campos por empresas o personas que se han enfocado en una de sus ramas de forma individual, derivándose en distintas subdivisiones que tratan diferentes métodos de la misma tecnología.

El estudio se centrará en las ventajas resultantes tras la utilización de la $\mathrm{AM}$ en la elaboración de placas de circuito impreso (AM PCB), donde se abordará la elaboración de dichas placas con esta tecnología y se procederá a compararlas con otros métodos anteriores a ella, como pueden ser la creación de placas electrónicas mediante la utilización de una fresadora a control numérico por computadora (CNC) que se conoce mundialmente como el método sustractivo, y definir cuáles son las ventajas que tengan una contra la otra. El estudio se orientó a este enfoque debido a la naturaleza compatible y complementaria entre una $\mathrm{CNC}$ y $\mathrm{AM}$, lo que

Digital Object Identifier (DOI):

http://dx.doi.org/10.18687/LACCEI2020.1.1.32

ISBN: 978-958-52071-4-1 ISSN: 2414-6390 significa que no necesitan y no debe ser mutuamente excluyentes [1].

Las tecnologías aditivas son una nueva puerta al futuro y entre las ventajas de la AM frente a los métodos de fabricación tradicional se encuentran la reducción considerable de desperdicio del material utilizado [2], fabricar en forma directa e inmediata, generar formas con geometrías complejas e irrealizables con tecnologías tradicionales [3], y es a partir de estas premisas que se orientará el estudio planteado a continuación y así analizar a fin de encontrar las distintas ventajas posibles y plasmarlas de manera que puedan ser identificadas cuando se haga referencia a la AM PCB.

A pesar de que algunas tecnologías AM, tales Como el proceso SL, comenzaron en el mercado hace muchos años, su investigación y desarrollo sigue en curso para tratar de afinar las diversas cualidades y ventajas que traen consigo todas estas tecnologías de AM [4]

El objetivo de este estudio es poder identificar cuáles son las ventajas puntuales de la utilización de la AM, para la implementación en la industria de las placas de circuito electrónico con respecto a otros métodos tradicionales.

\section{Sustento TEORICO}

El interés en la fabricación aditiva para la producción industrial surge ya que ofrece un futuro prometedor debido a las ventajas que traen consigo cada una de las técnicas y procesos de fabricación con respecto a su contra parte de procesos tradicionales, entre todas estas ventajas podemos encontrar la capacidad de producir estructuras más complejas, menos desperdicio de material, una configuración más rápida debido al procesamiento digital a digital y productos de menor peso. Para poder entender y retener mayor información sobre el tema tratado en esta sección es importante conocer que cuando hablemos de AM nos referimos a un proceso giratorio automatizado que se desarrolla a partir de un principio tecnológico basado en capas [5].

En una iniciativa en conjunto para la estandarización y normalización de la AM por parte de la ASTM y la ISO se presenta una propuesta donde reúnen este campo de estudio en siete categorías principales: VAT Photopolymerization, Powder Bed Fusion (PBF), Binder Jetting, Material Jetting, Sheet Lamination, Material Extrusion, Directed Energy Deposition (DED) [6], clasificación a la que se adjuntó una nueva categoría creada por Hybrid Manufacturing Technology compañía pionera en la implementación de la tecnología aditiva combinándola con métodos como milling $\mathrm{CNC}$, y es ella quien realizo la combinación de estas tecnologías en un hibrido, al que denomino como Hybrid o AMBIT permitiendo la combinación de maquinado CNC y deposición de metales 
por láser, este hecho es importante porque nos facilita la $\mathrm{AM}$ y sustractiva en una misma máquina [7].

Alrededor del mundo son muchos los países que se dedican al desarrollo de las tecnologías aditivas entre los que encontramos como referentes en Europa a España, país en donde al hablar de tecnología AM, de manera automática dirigimos nuestra atención hacia ADDIMAT(Asociación Española de Tecnologías de Fabricación Aditiva y 3D), esta es una asociación española que representa la $\mathrm{AM}$ en este país y para ello ADDIMAT integra a empresas de toda la cadena de valor de la fabricación aditiva e impresión 3D, siendo la primera en Europa en lograr reunir empresas de todo su país con el fin de desarrollar esta tecnología tan prometedora y con el fin de potenciar la colaboración, creando oportunidades de networking entre los diferentes agentes del mercado, en Alemania para el año 2016, la facturación total del sector de AM en Alemania habría sido como mínimo de 828 millones de euros (este dato es conservador, ya que sólo se computan las empresas dedicadas exclusivamente a la AM). Comparando este valor con la facturación global del sector en el mismo año (aproximadamente 5.462 millones de euros), en Alemania se habría generado como mínimo un $15,16 \%$ de la facturación a nivel global [8], se estima que los fabricantes alemanes están especializados en la rama industrial de la AM, y destacan en las áreas tecnológicas basadas en la fusión de lecho de polvo (PBF) y concretamente en las tecnologías SLM y SLS con polvo metálico. Empresas como EOS, Concept Laser (adquirida por General Electric en 2016 para formar parte del grupo GE Additive) y SLM Solutions son líderes mundiales en este campo [9]. Es muy importante hacer referencia que en el sector de la AM orientada a la electrónica en Europa, el país con el desarrollo más alto es Francia que, junto a Alemania, España y Reino Unido, se centran en la electrónica impresa y el desarrollo de procesos y materiales de aplicaciones versátiles [10].

En estados unidos la AM está en su mayor apogeo, la NASA está invirtiendo en la AM pues estas investigaciones prometen ventajas para futuros prototipos de naves espaciales, pero es el caso de cientos de eventos que catapultan esta tecnología lo que hace de estados unidos un lugar para desarrollarse en este sector, conferencias reconocida internacionalmente como AMUG 2020, o IMTS 2020, representados por la asociación de tecnología de manufactura(AMT) de ese país, son referencias claves de su alto desarrollo en esta área, empresas como Voltera, que son gigantes consolidados en cuanto a la presentación de prototipos híbridos ganando diversos premios nacionales e internacionales por su constante compromiso con el avance tecnológico y quienes presentan mejoras a sus prototipos muy frecuentemente tomando lo mejor de ambas tecnologías la aditiva y la sustractiva, con todos estas empresas dispuestas a invertir en tecnologías nuevas e investigaciones para el desarrollo de equipos novedosos que brinden soluciones prácticas y de la mano de la tecnología aditiva es imposible no pensar que este país una verdadera alma mater para esta tecnología.
Para nuestros fines orientaremos esta investigación en el estudio de las ventajas que trae consigo la utilización de AM, orientado a la impresión de placas de circuito impreso (PCB), y pasamos a elegir este tema de investigación considerando que todo dispositivo tecnológico en la actualidad cuenta con una placa electrónica por ende es importante analizar todas las posibilidades de mejora que hay disponibles en nuestro entorno para este sector tecnológico tan importante. El objetivo principal de este estudio es descubrir las ventajas que trae consigo la AM en su utilización para crear placas de circuito impreso, y es aquí donde nos centraremos y hablaremos de las disciplinas de la AM que se orienten a este fin. Se afirma que la AM es típicamente más lenta al procesar un material que su contraparte por mecanizado a control numérico por computadora [11].

Es importante saber todas estas ventajas pues cuando se maquina un circuito electrónico en baquelita se desperdicia mucha materia prima, tanto la que queda aislada en la misma placa luego de diseñar las pistas, como la que se remueve para maquinar el circuito pues se hace mediante el método sustractivo mientras que la AM tiene la ventaja de desperdicio "bajo cero" [12].

Al poder observar una placa generada por el método de fresado CNC se puede notar las cantidades de cobre que se desperdician, es importante reconocer que es un método confiable y estandarizado en muchas industrias y esto es precisamente porque tiene ventajas: precisión, simulación de procesos, confiabilidad, reducción de error humano y reducción de desperdicios con respecto a otros métodos artesanales, con la particularidad de que el material a utilizar se limita en la mayoría de los casos a baquelitas de cobre. La implementación de máquinas $\mathrm{CNC}$ para la fabricación de $\mathrm{PCB}$ con pistas con un mínimo de hasta $0.4 \mathrm{~mm}$ son factibles [13]. No obstante, al referirse a la AM se encuentran equipos híbridos que traen consigo precisión y detalle, al realizar todo el diseño por computadora se limita el error humano que antes se cometía al perforar y se reduce el límite que nos presentaban fresadoras $\mathrm{CNC}$ en cuanto a tamaños mínimos de las pistas, también hay mayores posibilidades para el desarrollo de los circuitos debido a que ya no estamos sujetos a imprimir sobre baquelita en una estructura plana y rígida solamente. "Se puede imprimir alrededor de curvas, en esferas $o$ en algo plano, o en una superficie flexible, que luego se puede doblar en la forma que se desee" [14].

Son muchas las posibilidades que brinda esta nueva tecnología dejando en el pasado la realización de placas electrónicas solamente en texturas rígidas bañadas en cobre, si abrimos nuestras mentes y trabajamos en ello podríamos obtener circuitos eléctricos en casi cualquier material y con casi cualquier forma posible. "Podemos hacer que estos cables tengan un ancho de micras", fueron palabras de Paquete científico de la NASA. "Estas líneas son muy pequeñas, de hasta 10 micras de ancho. Estos tamaños no son posibles utilizando los procesos tradicionales de fabricación de placas de circuito"[14]. 
Sabemos que los países que lideran el sector de la AM PCB son europeos como Francia y Alemania, también EE. UU, con algunas empresas como HYBRID, que cada día están generando nuevos equipos capaces de crear circuitos electrónicos más precisos. Es nuestro deber aprender a trabajar con estos equipos, si se deja que esta tecnología pase a y solo se observa como los demás avanzan con ella, seguiremos estancados en el pasado en procesos de hace más de 3 décadas, sin aprovechar, los recursos y el talento que tenemos a nuestro alcance. El equipo de HYBRID no solo trae las ventajas de la AM y el maquinado CNC en un solo dispositivo, sino que está adaptado a la tecnología de montaje superficial(SMT), lo que significa que en la placa no será necesariamente perforada, podría montar los componentes y dejarlos fundirse a la placa, utilizando las palabras de la propia página de Voltera, esta tecnología surge para la automatización industrial pero ha seguido su desarrollo a lo largo de las décadas y presenta una ventaja más, pues al perforar las pistas existe el riesgo de levantamiento de pistas en muchos de los casos, pero con la implementación de esta tecnología se evita por completo este error aunque para ello se tiene que utilizar los dispositivos de ensamblaje superficial adecuados.

\section{A. Materiales y metodologia}

En este estudio se utilizará como herramienta para las pruebas con el método aditivo una Voltera V-ONE, esta cuenta con una plataforma de impresión y Materiales de impresión trabajando con cartuchos de $2 \mathrm{ml}$ de material de impresión, tinta conductora y pasta de soldadura [15]. El software utilizado es el que brinda la misma compañía Voltera, dentro de sus funciones estas las de; imprimir, soldar, calor y taladro.

Una de las ventajas de utilizar este equipo es su versatilidad en el momento de la creación del circuito ya que se puede controlar el ancho de las líneas mientras se está trabajando. Otra de las ventajas es que con este equipo se puede colocar la pasta para la soldadura en el mismo circuito. Los archivos que este software puede operar son todos aquellos con extensión gerber, normalmente generados por software de diseño de circuitos eléctricos como lo son Proteus, Eagle, KidCAD, Altium.

Esta investigación tendrá un enfoque mixto pues el principal objetivo es determinar las ventajas y cualidades de la AM para la industria de producción de circuitos electrónicos, y a su vez cuantitativo porque se busca demostrar el porcentaje de ahorro de materia prima con respecto a su método predecesor realizando las pruebas que sean necesarias para comprobar la premisa.
A continuación, se muestran las variables a considerar en nuestro estudio:

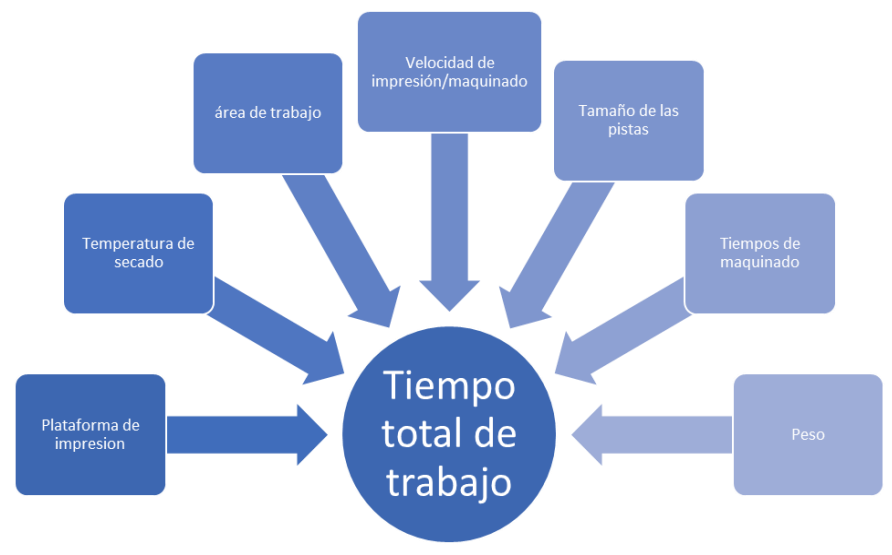

Fig. 1. Variables de investigación. Fuente: Creación Propia.

Este estudio se realizará mediante pruebas, diseñando placas electrónicas básicas e imprimiéndolas con la Voltera, luego realizando el mismo proceso con la fresadora CNC y se proseguirá con un proceso de comparación de resultados obtenidos en la etapa anterior para realizar un análisis. Cada circuito fue diseñado primeramente en Proteus, donde se creó el archivo PCB, que posteriormente fue derivado en dos opciones, primero, la capa con extensión. GBR, específicamente la capa CADCAM BOTTOM COPPER, fue destinada para ser procesada en la V-One de Voltera ya que es en esta capa donde se procesan las pistas del circuito. Segundo, la etapa de fresado CNC, esta fue seleccionada luego de obtener el PCB la misma capa, paso por el programa COPPERCAM donde se definieron los atributos necesarios para maquinar el circuito y luego paso por el software CNC CHANGER para adecuar su extensión y ser procesada por las fresadoras $\mathrm{CNC}$ a disposición. Finalmente se detallan las tablas de resultados de los circuitos realizados mediante el método aditivo con la V-One de Voltera, describiendo cada uno de los procesos.

\section{B. Resultados}

A continuación, se mostrarán los datos obtenidos a lo largo de la investigación los cuales fueron recopilados y agrupados en tablas que posteriormente discutiremos cada uno de los campos obtenidos tanto en el método aditivo como en el sustractivo. 
TABLA I

Pruebas en Voltera V-One. TeCNOlogía Aditiva. Fuente: Creación Propia

\begin{tabular}{|c|c|c|c|c|c|c|c|c|c|c|c|}
\hline \# & Material & $\begin{array}{c}\text { Dimensione } \\
\text { s } \\
\text { de la placa }\end{array}$ & $\begin{array}{c}\text { Tamaño } \\
\text { de las } \\
\text { pistas }\end{array}$ & $\begin{array}{c}\text { Peso } \\
\text { antes de } \\
\text { imprimir }\end{array}$ & $\begin{array}{c}\text { Peso } \\
\text { después } \\
\text { de } \\
\text { imprimir }\end{array}$ & $\begin{array}{c}\text { Temperatur } \\
\text { a } \\
\text { De secado }\end{array}$ & $\begin{array}{c}\text { Veloci } \\
\text { dad }\end{array}$ & $\begin{array}{c}\text { Tiempo } \\
\text { de } \\
\text { calibració } \\
\mathrm{n}\end{array}$ & $\begin{array}{c}\text { Tie } \\
\text { mpo } \\
\text { de } \\
\text { Imp } \\
\text { resi } \\
\text { ón }\end{array}$ & $\begin{array}{l}\text { Tiempo de } \\
\text { secado }\end{array}$ & $\begin{array}{c}\text { Tiempo de } \\
\text { trabajo } \\
\text { aproximado }\end{array}$ \\
\hline 1 & $\begin{array}{c}\text { Baquelita } \\
\text { Voltera }\end{array}$ & $109 * 83 \mathrm{~mm}$ & $1.35 \mathrm{~mm}$ & $28.5 \mathrm{~g}$ & $28.6 \mathrm{~g}$ & $210^{\circ} \mathrm{C}$ & $\begin{array}{l}10000 \\
\text { RPM }\end{array}$ & $\begin{array}{c}\text { 7:00 min } \\
\text { aprox }\end{array}$ & 29:05 min & $60 \mathrm{~min}$ & $97 \mathrm{~min}$ \\
\hline 2 & $\begin{array}{c}\text { Baquelita } \\
\text { Voltera }\end{array}$ & $83 * 58 \mathrm{~mm}$ & $0.4 \mathrm{~mm}$ & $15.3 \mathrm{~g}$ & 15.4 & $210^{\circ} \mathrm{C}$ & $\begin{array}{l}10000 \\
\text { RPM }\end{array}$ & $2 \mathrm{~min}$ & 6:00 min & $\begin{array}{c}180 \text { min } \\
\text { (temperatur } \\
\text { a ambiente) } \\
\text { en secado } \\
\text { normal } \\
\text { serian } 40 \\
\text { min }\end{array}$ & $\begin{array}{c}188 \text { min } \\
\text { En secado } \\
\text { normal } 48 \\
\text { min }\end{array}$ \\
\hline 3 & $\begin{array}{c}\text { Baquelita } \\
\text { Voltera }\end{array}$ & $83 * 58 \mathrm{~mm}$ & $2 \mathrm{~mm}$ & $15 \mathrm{~g}$ & 15.3 & $210^{\circ} \mathrm{C}$ & $\begin{array}{l}10000 \\
\text { RPM }\end{array}$ & $2: 46 \min$ & $7.20 \mathrm{~min}$ & $\begin{array}{c}180 \text { min } \\
\text { (secado al } \\
\text { ambiente) } \\
\text { en secado } \\
\text { normal } \\
\text { serian } 40 \\
\text { min } \\
\end{array}$ & $\begin{array}{l}190 \text { min En } \\
\text { secado } \\
\text { normal } 50 \\
\quad \text { min }\end{array}$ \\
\hline 4 & Madera & $\begin{array}{c}133.5 * 100 \\
\mathrm{~mm}\end{array}$ & $1.35 \mathrm{~mm}$ & N.P & N.P & $210^{\circ} \mathrm{C}$ & $\begin{array}{l}10000 \\
\text { RPM }\end{array}$ & 1:07 min & $3: 40 \mathrm{~min}$ & $46 \mathrm{~min}$ & $51 \mathrm{~min}$ \\
\hline 3 & Acrílico & $99 * 68 \mathrm{~mm}$ & $2 \mathrm{~mm}$ & 31.6 & 31.7 & $210^{\circ} \mathrm{C}$ & $\begin{array}{l}10000 \\
\text { RPM }\end{array}$ & $2: 46 \min$ & $7: 20 \mathrm{~min}$ & $38 \mathrm{~min}$ & $48 \mathrm{~min}$ \\
\hline 4 & Cartón & $99 * 60 \mathrm{~mm}$ & $1.35 \mathrm{~mm}$ & N.P & N.P & $210^{\circ} \mathrm{C}$ & $\begin{array}{l}10000 \\
\text { RPM }\end{array}$ & 1:07 min & 3:40 min & $25 \mathrm{~min}$ & $30 \mathrm{~min}$ \\
\hline 5 & $\begin{array}{c}\text { Baquelita } \\
\text { Voltera }\end{array}$ & $\begin{array}{c}\text { Reutilizació } \\
\mathrm{n} \text { de } \\
\text { espacio }\end{array}$ & $1 \mathrm{~mm}$ & $15.5 \mathrm{~g}$ & $15.6 \mathrm{~g}$ & $210^{\circ} \mathrm{C}$ & $\begin{array}{l}10000 \\
\text { RPM }\end{array}$ & $20 \mathrm{seg}$ & $1: 10 \mathrm{~min}$ & $23 \mathrm{~min}$ & $25 \mathrm{~min}$ \\
\hline
\end{tabular}

Para la parte aditiva se trabajó realizando circuitos de los cuales resaltamos los siguientes:
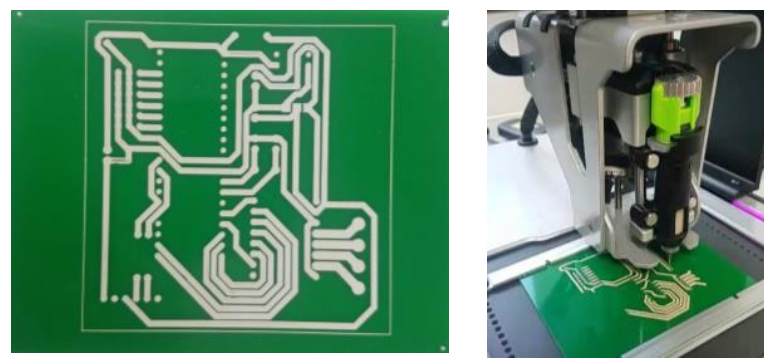

Fig. 2 y 3. Circuito1 AM PCB. Fuente: Creación Propia.

El circuito anterior fue diseñado en Proteus, maquinado por la Voltera mediante el método aditivo, luego de ser generado se procedió con el secado, a una temperatura de 210 ${ }^{\circ} \mathrm{C}$, durante un tiempo de 60 minutos hasta generar un acabado como el que se muestra en la figura 2 y 3 . El tiempo aproximado de trabajo en esta placa fue de 107 minutos, tomando en cuenta los 97 de trabajo de la maquina y los tiempos gastados en ubicar la placa dentro de la misma y repetir el proceso girándola para su secado.
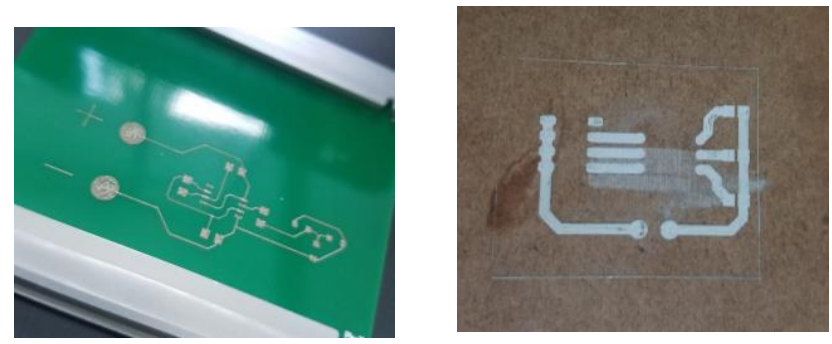

Fig. 4. Circuito $0.4 \mathrm{~mm}$ pistas 1, Fig. 5. Circuito Madera.

Fuente: Creación Propia.

El circuito de la figura 4 es el modelo de ejemplo proporcionado por VOLTERA, denominado circuito HELLO WORLD, este fue tomado de los ejemplos brindados por el servidor de la empresa, fue maquinado en un total de 188 minutos en secado natural, y en el secado propuesto por el proveedor del equipo un tiempo aproximado de $48 \mathrm{~min}$, este circuito tiene un dato interesante y es el tamaño de sus pistas el cual es de $0.4 \mathrm{~mm}$. En el circuito de la figura 5 , se resalta la versatilidad de plataforma de impresión, para este circuito se utilizó una placa de madera sobre la que se imprimió el circuito, en un tiempo aproximado de 3 minutos con 40 segundos. 


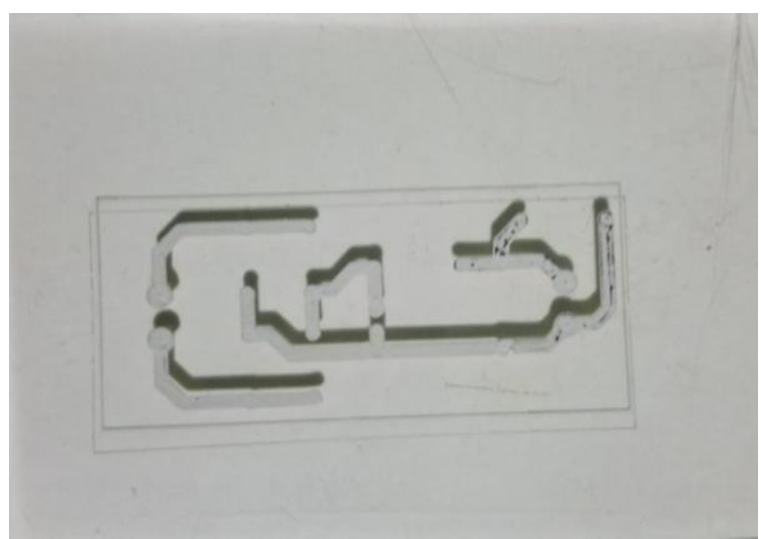

Fig. 6. Circuito Acrílico. Fuente: Creación Propia.

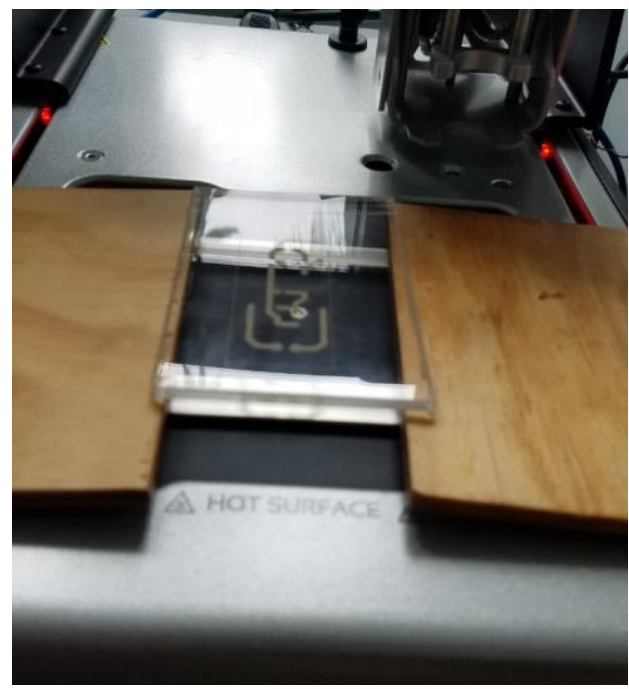

Fig.7. Secado. Fuente: Creación Propia.

La figura 6 nos muestra un circuito repetido con la variante que fue trabajado sobre acrílico como plataforma de impresión, y mejoró notablemente el tiempo de secado de 38 minutos, obteniendo un circuito funcional según la prueba de conductividad, cabe mencionar que el acrílico se deforma al contacto con altas temperaturas, por lo que es necesario retirarlo a los 20 minutos de la superficie caliente, dejarlo secar en temperatura ambiente o estarlo levantando de la plataforma caliente en lapsos de entre 10 minutos.

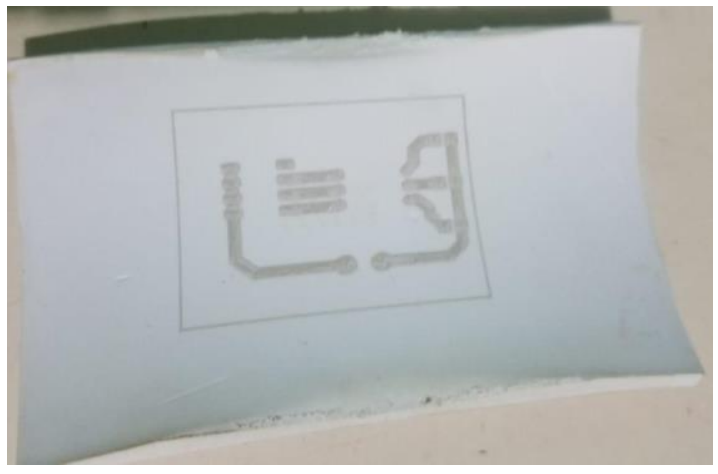

Fig. 8. Circuito Cartón. Fuente: Creación Propia.

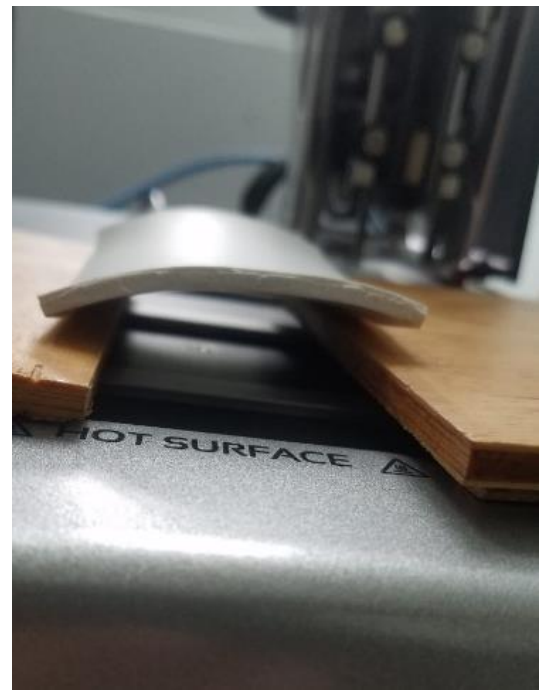

Fig. 9. Deformación. Fuente: Creación Propia

La figura 8 muestra el circuito propuesto, maquinado sobre cartón, en una de las diversas pruebas para comprobar plataformas de impresión, es lamentable apuntar que la rigidez del cartón llego a un punto de deformación alto, a los 25 minutos de estar en proceso de secado se tuvo que retirar de la plataforma. Al realizar las pruebas de continuidad del circuito fueron positivas, el circuito funciono a la perfección aun después de la curvatura tomada. Es recomendable para realizar circuitos en cartón realizar un secado natural. 
TABLA 2

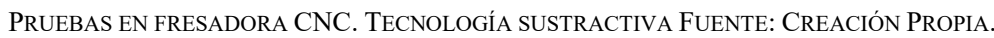

\begin{tabular}{|c|c|c|c|c|c|c|c|c|c|}
\hline$\#$ & Material & $\begin{array}{c}\text { Dimensiones } \\
\text { De la placa }\end{array}$ & $\begin{array}{c}\text { Tamaño de } \\
\text { las pistas }\end{array}$ & $\begin{array}{c}\text { Peso antes } \\
\text { de } \\
\text { maquinado }\end{array}$ & $\begin{array}{c}\text { Peso } \\
\text { despues de } \\
\text { maquinado }\end{array}$ & Velocidad & $\begin{array}{c}\text { Tiempo de } \\
\text { calibracion }\end{array}$ & $\begin{array}{c}\text { Tiempo de } \\
\text { maquinado }\end{array}$ & $\begin{array}{c}\text { Tiempo } \\
\text { de } \\
\text { trabajo }\end{array}$ \\
\hline 1 & $\begin{array}{c}\text { Baquelita } \\
\text { cobre }\end{array}$ & $75 * 75 \mathrm{~mm}$ & $1.3 \mathrm{~mm}$ & $13 \mathrm{~g}$ & $10.4 \mathrm{~g}$ & $\begin{array}{c}12000 \\
\text { RPM }\end{array}$ & $1 \mathrm{~min}$ & $8: 52 \mathrm{~min}$ & $10 \mathrm{~min}$ \\
\hline 2 & $\begin{array}{c}\text { Baquelita } \\
\text { cobre }\end{array}$ & $72 * 46 \mathrm{~mm}$ & $0.4 \mathrm{~mm}$ & $6.9 \mathrm{~g}$ & $6.6 \mathrm{~g}$ & $\begin{array}{c}12000 \\
\mathrm{RPM}\end{array}$ & $1 \mathrm{~min}$ & $2: 49 \mathrm{~min}$ & $3: 49 \mathrm{~min}$ \\
\hline 3 & $\begin{array}{c}\text { Baquelita } \\
\text { cobre }\end{array}$ & $65 * 30 \mathrm{~mm}$ & $2 \mathrm{~mm}$ & $5 \mathrm{~g}$ & $3.6 \mathrm{~g}$ & $\begin{array}{c}12000 \\
\mathrm{RPM}\end{array}$ & $1 \mathrm{~min}$ & $3 \mathrm{~min}$ & $4 \mathrm{~min}$ \\
\hline 5 & $\begin{array}{c}\text { Baquelita } \\
\text { cobre }\end{array}$ & $46 * 36 \mathrm{~mm}$ & $1.35 \mathrm{~mm}$ & $4 \mathrm{~g}$ & $3.1 \mathrm{~g}$ & $\begin{array}{c}12000 \\
\mathrm{RPM}\end{array}$ & $1 \mathrm{~min}$ & $2: 10 \mathrm{~min}$ & $3: 10 \mathrm{~min}$ \\
\hline cobre & $21 * 20 \mathrm{~mm}$ & $1 \mathrm{~mm}$ & $0.9 \mathrm{~g}$ & $0.8 \mathrm{~g}$ & $\begin{array}{c}12000 \\
\mathrm{RPM}\end{array}$ & $1 \mathrm{~min}$ & $1 \mathrm{~min}$ & $2 \mathrm{~min}$ \\
\hline
\end{tabular}

En el circuito mostrado en la figura 10 se puede determinar que funcionará, con un tiempo de procesamiento

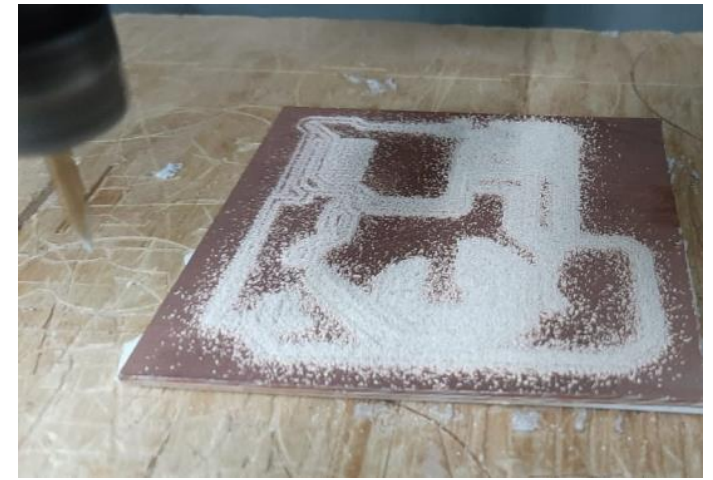

Fig. 10. Circuito1. Fuente: Creación Propia

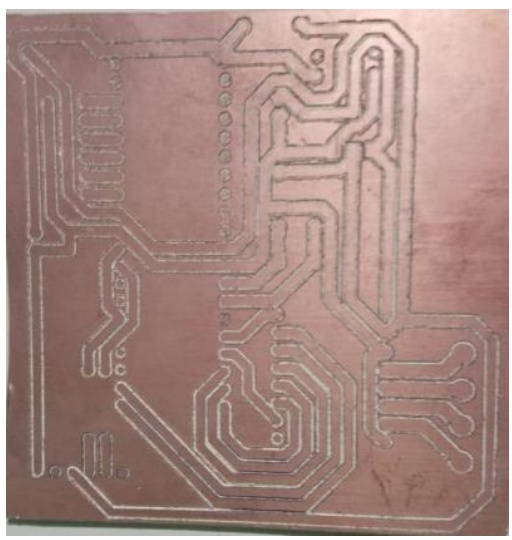

Fig. 11. Tecnología sustractiva. Fuente: Creación Propia. de 10 minutos. Además de ello es interesante el hecho de que el tamaño de las pistas no fue problemático, con la única observación que se deberá proceder con cuidado al momento de realizar el proceso de soldadura pues la poca destreza con el cautín podría desembocar en un corto circuito. Es importante mencionar que este circuito luego de ser maquinado sufrió una pérdida de peso de $2.6 \mathrm{~g}$.

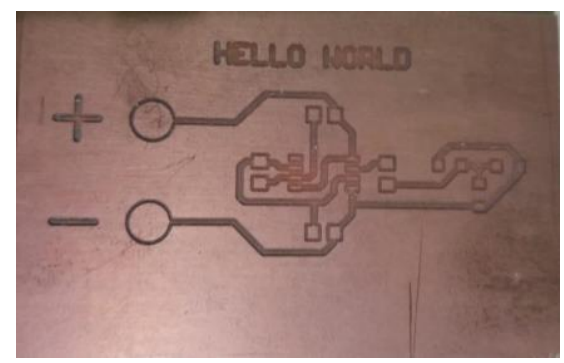

Fig. 12. Circuito Hello world. Fuente: Creación Propia.

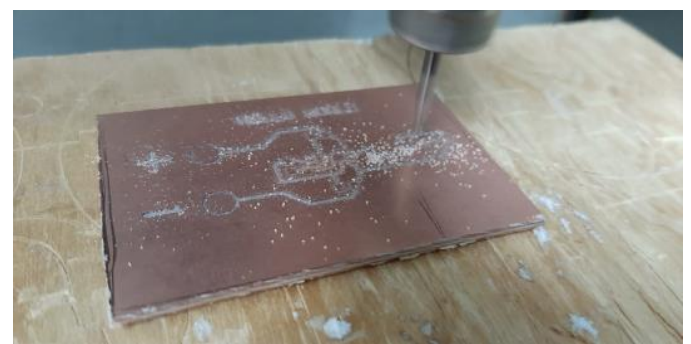

Fig. 13. Maquinado. Fuente: Creación Propia 
Este circuito es muy importante, debido a que se utilizaron pistas de $0.4 \mathrm{~mm}$, aun utilizando una fresa especial, con 15 grados, para tener una punta fina y poder maquinar las pistas más pequeñas, pero, aunque se tomaron las medidas para lograr su funcionamiento adecuado, el circuito no tiene continuidad en diversos puntos de este. A su vez soldar con estaño los componentes se volvería una tarea tediosa.

\section{Discucion}

A continuación, se analizan las variables independientes de la investigación, comparando su efecto en el proceso aditivo y sustractivo, para poder entender la viabilidad de esta tecnología en la industria.

1) Plataforma de impresión: En base a los resultados expuestos anteriormente, se afirma que la AM puede maquinar circuitos sobre acrílico con toda confianza siempre que mantengamos un control manual para la temperatura, del mismo modo pasa con el cristal y la madera, facilitando el proceso de creación de cualquier circuito.

Se pudo observar a lo largo de los resultados que la impresión de cualquier circuito es perfectamente viable en plataformas como las utilizadas, siempre y cuando la superficie sea plana y sin rugosidades que interfieran con el equipo, claro está que el problema en este punto es el paso del secado, por lo que sería mejor realizar un secado natural o mantener el control de la temperatura por pequeños periodos de tiempo pausados, para evitar que los materiales utilizados se deformen por las altas temperaturas como sucedió con el circuito que fue impreso en cartón, el cual llego a tener una curvatura muy alta, y aunque el circuito seguía aprobando las pruebas de continuidad lamentablemente fue descartado debido a las deformaciones sufridas a lo largo del proceso. El método sustractivo no cuenta una variedad de plataformas de impresión como lo muestra la AM.

2) Temperatura de secado: En esta parte del análisis y en base a los resultados obtenidos podemos hacer las siguientes afirmaciones, el método sustractivo no utiliza tiempo de trabajo en secado del circuito, así que hay un ahorro de tiempo considerable.

En tiempo de secado del método aditivo es afectado por el tipo de tinta conductora que estemos utilizando, y la temperatura a la que estemos trabajando. El circuito impreso en baquelita de Voltera, en la prueba 5, con tan solo 23 minutos de calor este ya estaba en condiciones de uso. Por ende, no es necesario secarlo durante 60 minutos a 210 grados.

De hecho, es factible reducir la temperatura y retirarlo algunos minutos antes, pero esto solo se puede determinar si se lleva un control constante del circuito y se llega a la conclusión que no es necesario mantenerlo más tiempo en secado.

3) Área de trabajo: El área de trabajo será subjetiva en ambos métodos, pues esta dependerá de la persona que trabaje el circuito, muchas personas prefieren tener un circuito con mucho espacio para evitar corto circuitos, otras prefieren los circuitos más pequeños. Las dimensiones de trabajo también dependen de la aplicación del circuito, dependiendo la utilidad que se le dará se deberá elegir un material más resistente o con más tamaño que otros.

4) Velocidad de impresión/maquinado: La velocidad es dependiente al equipo que estemos utilizando para realizar el trabajo, por ejemplo, si elegimos una fresadora CNC, se debe saber que motores se tienen instalados en el equipo y que numero de revoluciones trae de fábrica. En el caso de la Voltera, la velocidad máxima que podría alcanzar el equipo que tenemos a disposición para este trabajo es de 10,000 rpm, en el caso de las fresadoras la velocidad máxima es de 12,000 rpm. Pero estos datos pueden variar dependiendo que equipo se utiliza, pero así que podría ser de mayor capacidad o menor.

5) Tamaño de las pistas: $\mathrm{Si}$ en una fresadora $\mathrm{CNC}$ queremos maquinar un circuito con pistas muy delgadas, procedemos a utilizar herramientas adecuadas, como una fresa de 10 grados, la cual nos sirve para maquinar de forma precisa, pero aun con estas herramientas el límite mecánico de la fresadora es de 0.4 $\mathrm{mm}$, como lo explicamos al inicio en este estudio. Cuando se trata de realizar circuitos de tamaños menores a estos se presentan fallas en el trabajo, como se visualiza en la figura 13. Por lo que no es una práctica recomendable crear circuitos de esta forma o al menos con esta tecnología.

Para la AM, en la Voltera, este paso todas las pruebas de continuidad y está claro que aun podemos reducir esta medida de las pistas. Con el equipo V-One de Voltera al igual que para el método sustractivo, la capacidad de generar pistas pequeñas dependerá en gran parte de las herramientas utilizadas, se hace referencia precisamente a las boquillas que se utilizan en el proceso, mientras la boquilla sea más pequeña la abertura de salida, más podremos trabajar con pistas pequeñas. Es indudablemente beneficioso la creación de circuitos que requieran poco espacio, pero siempre recordando que la debilidad de estos circuitos en la parte de potencia, son circuitos más orientados a prototipos.

6) Tiempo de maquinado: Los tiempos de maquinado son el principal punto de quiebre para la tecnología aditiva, pues un circuito toma mucho tiempo y no por su maquinado sino precisamente en la etapa de secado. Y aquí es donde no podríamos ocultar este hecho pues si deseamos realizar una producción a gran escala, este método puede ser deficiente.

Lo anteriormente mencionado se puede notar al comprarlo entre ambos métodos, para conocer qué porcentaje de tiempo se tarda más un proceso que el otro procederemos a realizar una tabla de comparación, en la que mostraremos los circuitos consultados, el tiempo utilizado en cada método, y el porcentaje de tiempo que tarda el maquinado de cada circuito. Para proceder utilizaremos la siguiente formula:

\% de tiempo: ((tiempo de trabajo fresado CNC) / (tiempo de trabajo Voltera)) $* 100$ 
TABLA 3.

DifERENCIAS DE TIEMPOS. FUENTE: CREACIÓN PROPIA.

\begin{tabular}{|c|c|c|c|}
\hline Circuito & $\begin{array}{c}\text { Tiempo de } \\
\text { trabajo Voltera }\end{array}$ & $\begin{array}{c}\text { Tiempo de trabajo } \\
\text { Fresadora CNC }\end{array}$ & \% de tiempo \\
\hline 1 & $97 \mathrm{~min}$ & $10 \mathrm{~min}$ & 10.3 \\
\hline 2 & $48 \mathrm{~min}$ & $4 \mathrm{~min}$ & 8.33 \\
\hline 3 & $50 \mathrm{~min}$ & $4 \mathrm{~min}$ & 8 \\
\hline 4 & $51 \mathrm{~min}$ & $3 \mathrm{~min}$ & 5.88 \\
\hline 5 & $25 \mathrm{~min}$ & $2 \mathrm{~min}$ & 8 \\
\hline
\end{tabular}

Se puede observar que la realización de un circuito en la fresadora CNC tomara aproximadamente entre un $8 \%$ y un $10 \%$ del tiempo total que se tardara el realizar el mismo circuito en la V-One de Voltera, si esta es trabajada con las instrucciones del fabricante. Estos tiempos podrían reducirse si se decide operar el tiempo de secado de forma manual, pero esto significaría estar completamente dedicado a al proceso.

De esta forma si lo que necesitamos es un circuito urgente la mejor forma de trabajarlo es mediante el método de fresado CNC. Pero esta parte es muy a discreción del usuario final.

7) Peso: Es importante conocer cuanta materia prima se utiliza en el método aditivo, y cuanta materia prima se desperdicia en el método sustractivo. En este estudio no entraremos en detalles como el costo de la materia prima en cada situación, sino que meramente en las cantidades que se desperdician de material. Para el caso de aditivo, mientras mayor sea la diferencia del peso en la superficie de trabajo será peor, y para el método sustractivo mientras menos peso tenga la superficie de trabajo será peor. De esta forma mostraremos las tablas respectivas de cada método, donde trabajaremos solamente con los pesos.

Para calcular el diferencial de peso en el método aditivo:

Diferencia $=($ peso final - Peso inicial $)$

Para calcular el diferencial de peso en el método sustractivo:

Diferencia $=($ peso inicial - peso final $)$

Estas fórmulas se tomaron de esta forma debido a que en el método aditivo el peso aumentará al final del trabajo, en cambio en el método sustractivo el acabado final tendrá menos peso que al comienzo.

TABLA 4.

DifERENCIAS DE PESOS. FueNTE: CREACIÓN PROPIA

\begin{tabular}{|c|c|c|c|c|c|c|}
\hline$\#$ & $\begin{array}{c}\text { Peso } \\
\text { Inicial } \\
\mathrm{V}\end{array}$ & $\begin{array}{c}\text { Peso } \\
\text { final V }\end{array}$ & Diferencia & $\begin{array}{c}\text { Peso } \\
\text { inicial F }\end{array}$ & $\begin{array}{c}\text { Peso } \\
\text { final F }\end{array}$ & Diferencia \\
\hline 1 & $28.4 \mathrm{~g}$ & $28.6 \mathrm{~g}$ & $0.4 \mathrm{~g}$ & $13 \mathrm{~g}$ & $10.4 \mathrm{~g}$ & $2.6 \mathrm{~g}$ \\
\hline 2 & $15.3 \mathrm{~g}$ & 15.4 & $0.1 \mathrm{~g}$ & $6.9 \mathrm{~g}$ & $6.6 \mathrm{~g}$ & $0.3 \mathrm{~g}$ \\
\hline 3 & $15 \mathrm{~g}$ & $15.3 \mathrm{~g}$ & $0.3 \mathrm{~g}$ & $5 \mathrm{~g}$ & $3.6 \mathrm{~g}$ & $1.4 \mathrm{~g}$ \\
\hline 5 & $15.5 \mathrm{~g}$ & $15.6 \mathrm{~g}$ & $0.1 \mathrm{~g}$ & $0.9 \mathrm{~g}$ & $0.8 \mathrm{~g}$ & $0.1 \mathrm{~g}$ \\
\hline
\end{tabular}

Como se observa en la tabla anterior al comparar los procesos de elaboración de los mismos circuitos en ambos métodos. En la elaboración del circuito del ejemplo número no mediante el método aditivo tendremos una ganancia de 0.4 gramos en el peso final, en cambio la elaboración del mismo circuito por el método de fresado CNC, se tendrá una pérdida de 2.6 gramos en el peso final del circuito. Nos enfrentamos a la misma situación en los siguientes ejemplos.

Según los datos obtenidos en la elaboración de los mismos circuitos en ambos procesos, en el método sustractivo se perderá una mayor cantidad de materia prima, en comparación a la que se gastará en el método aditivo. Esto se debe a que en el método aditivo el flujo de tinta no es muy alto, solo es ininterrumpido, por ende, crea capas con una densidad uniforme, y es de esa forma que los circuitos son conductores, sin necesidad de rellenar la placa de tinta conductora, en cambio el método sustractivo por su misma forma de trabajo removerá materia prima indiscriminadamente a través de su paso por el sustrato utilizado.

Otras ventajas que es importante mencionar, es que en el método aditivo no tenemos piezas que se quiebren porque no existe una fricción entre la baquelita y la herramienta, contrario a las fresadoras $\mathrm{CNC}$, que al estar en contacto la herramienta con la pieza, hay un desgaste y muchas veces quebraduras, este fenómeno desata otro y seria que al quebrarse una fresa, es dificultoso continuar trabajando el mismo circuito, o si la pieza está a desnivel será difícil continuar con el mismo circuito, caso similar sucede si hay un corte de energía y no se ha terminado una pieza, en la mayoría de ocasiones tendremos que usar otra baquelita y comenzar de cero el circuito. En cambio en el método aditivo, las herramientas no se quiebran porque no hacen contacto con la pieza, si se pueden atascar pero solo se limpian y se pueden continuar usando, por otra parte si nuestro circuito está a desnivel, o si se va la energía y no se terminó un trabajo en proceso, cuando estemos preparados para continuar con el trabajo podremos continuar a partir de donde nos quedamos, esto porque estos sistemas híbridos tienen la particular ventaja de seleccionar por donde nos quedamos y continuar a partir de ahí, o si incluso una parte del circuito no cumplió sus expectativas o falto una parte de su acabado simplemente se seleccionan las pistas necesarias y automáticamente vuelve a repasar solamente las pistas seleccionadas, y no existe algún problema si removemos el circuito pues el equipo de Voltera tiene su propio sistema para detectar el circuito que se trabajó

\section{CONCLUSIONES}

- Se identifico que las ventajas que sobresalen en la manufactura aditiva son principalmente la diversidad de plataformas en las que podemos trabajar, así como el tamaño de las pistas que puede ser realmente pequeño, y su principal desventaja el tiempo de trabajo contrario a su contraparte en el método sustractivo. Siendo esta tecnología ideal su implementación en el desarrollo de proyectos para robots con reducidos espacios de trabajo[16].

- Se analizaron los procesos de AM PCB y fresado $\mathrm{CNC}$ logrando identificar diferencias notables como 
la posibilidad de utilizar diferentes sustratos en el método aditivo, lo cual es imposible en el método sustractivo.

- Se definió que cada método de manufactura de PCB es útil dependiendo de la situación experimentada, y se logró ver las ventajas de cada cual en primera persona y con respecto a estos resultados se logró seleccionar cual método es más convenientes según lo requiera el trabajo a realizar, y para la industria se sugiere el método sustractivo que sigue siendo en la actualidad el más ventajoso.

- Se demostró la eficiencia del método AM PCB, cada ventaja ofrecida por esta nueva tecnología y como explotarla en diferentes proyectos, pero su más grande desventaja el tiempo de trabajo sigue siendo uno de los problemas a resolver para ser totalmente eficiente y poder ser implementada en procesos industriales de producción a gran escala.

\section{REFERENCES}

[1] J. B. Jones, «The Synergies of Hybridizing CNC and Additive Manufacturing», 2014.

[2] A. Balbas Calvo, M. del M. Espinoza, y M. Domínguez Somonte, «últimos avances en la fabricación aditiva con materiales metálicos», Lampsakos, vol. N. 19, pp. 47-54, 2018. DOI: http://dx.doi.org/10.21501/21454086.2365

[3] D. Torreblanca Diaz, «Tecnologías de Fabricación Digital Aditiva, ventajas para la construcción de modelos, prototipos y series cortas en el proceso de diseño de productos», ICONOFACTO, vol. Vol. 12 N. 18, pp. 118-143, 2016. DOI: http://dx.doi.org/10.18566/v12n18.a07

[4] F. Calignano, D. Manfredi, E. P. Ambrosio, S. Biamino, M. Lombardi, y E. Atzeni, «Overview on Additive Manufacturing Technologies», IEEE, vol. Vol. 105, 2017.

[5] A. Gebhardt, Understanding Additive Manufacturing. Alemania, Munich: Hanser Publications, 2011.

[6] ISO y ASTM, «ISO/TC 261 and ASTM F42», vol. 1, 2015.

[7] ]Hybrid manufacturing technologies, «7 families of additive manufacturing», ISO/ASTM52900-15, 2015.

[8] J. L. Peralta Herman, «El mercado de la Fabricación Aditiva en Alemania», Bajo la supervisión de la Oficina Económica y Comercial de la Embajada de España en Berlín., 2016.

[9] T. Grimm, B. Günther, D. Bauer, y K. Borchers, «Additive Fertigungsverfahren 2016 - Handlungsfelder», VDI Verein Deutscher Ingenieure e.V. Abril 2016, 2016.

[10] AMEF2018, «A Strategic approach to increasing Europe's value proposition for additive manufacturing technologies and capabilities.», 2016. .

[11] J. B. Jones, «Repurposing mainstream CNC machine tools for laserbased additive manufacturing», Proc. of SPIE, vol. Vol. 9738, 2016. DOI: https://doi.org/10.1117/12.2217901

[12][12] V. R. Manda, V. Kampurath, y C. Msrk, «3D printing and its effect on outsourcing: A study of the Indian aircraft industry», J. Aerosp. Technol. Manag., 2018. DOI: http://dx.doi.org/10.5028/jatm.v10.862

[13] R. S. Guadrón Gutiérrez y J. J. Guevara Vásquez, «Experiencias en la Automatización de la Construcción de Circuitos Impresos con Máquina CNC de Código Abierto», Rev. Tecnol. Esc. Espec. En Ing. ITCAFEPADE, vol. 8, 2015.

[14] L. Keesey, «NASA Investigates 3-D Printing for Building Densely Populated Electronic Assemblies», Space Tech, 2017. .

[15] J. Zozaya, «Voltera | Build Hardware Faster», Voltera V-One. Prototyping tool., 2018. [En línea]. Disponible en: https://www.voltera.io. [Accedido: 23-nov-2019].
[16] J. L. Ávila, H. Jiménez, T. Márquez, C. Muñoz, D. Nolasco, D. Miselem, M. Perdomo, M. Carrasco «Teleoperated voice picking robots prototype as a logistic solution in Honduras», IEEE, 2020,

DOI: https://doi.org/10.1109/ICCRE49379.2020.9096483 